\title{
Incidence and prognostic factors of hepatoid adenocarcinoma: a population-based analysis
}

\author{
Wenting Wang, Guang Li \\ Department of Radiation Oncology, The First Hospital of China Medical University, Shenyang, China \\ Contributions: (I) Conception and design: W Wang; (II) Administrative support: G Li; (III) Provision of study materials or patients: W Wang; (IV) \\ Collection and assembly of data: W Wang; (V) Data analysis and interpretation: W Wang; (VI) Manuscript writing: All authors; (VII) Final approval \\ of manuscript: All authors. \\ Correspondence to: Guang Li, MD. Department of Radiation Oncology, The First Hospital of China Medical University, 155 Nanjing North Street, \\ Shenyang, China. Email cmuliguang@163.com.
}

\begin{abstract}
Background: Hepatoid adenocarcinoma (HAC) is a kind of rare extrahepatic adenocarcinoma with hepatocyte differentiation. At present, the incidence and prognostic factors of HAC remain poorly discussed. Methods: We extracted two cohorts from Surveillance, Epidemiology, and End Results (SEER) database by restricting the histology to HAC. One cohort of 258 eligible patients was from SEER 21 Regs Limited-Field Research Data + Hurricane Katrina Impacted Louisiana Cases [2000-2016] to compute incidence, and the other cohort of 121 eligible patients was from SEER 18 Regs Custom Data (with additional treatment fields) [1975-2016] to estimate survival and risk factors. The incidence wad computed by the Joinpoint Regression Program (Version 4.7.0.0). The overall survival (OS) and prognostic factors were analyzed by univariate and multivariate analyses in SPSS 24.0.
\end{abstract}

Results: The overall incidence of HAC between 2000 and 2016 was 0.014 per 100,000 people (95\% CI: 0.012 to 0.015 ). The most common primary site was the lung, followed by the digestive, urinary and reproductive organs, but in the previously published case reports, the stomach was the most common site. The median age at diagnosis was 66 (range, 31-85) years. A total of $28.1 \%$ of patients underwent surgery, while $71.9 \%$ underwent radiotherapy or/and chemotherapy. The 1-year survival rate of these patients was $35.0 \%$, and the 3 -year survival rate was only $16.9 \%$. The median survival time was 5 months (95\% CI: 2.97 to 7.03 months). Multivariate analysis showed that age less than 60 years, no distant metastasis, surgery and chemotherapy were independent prognostic factors related to a better outcome.

Conclusions: This study suggests that HAC is a rare malignant tumor with poor prognosis, and its most common site is lung. Its incidence is increasing year by year. Age at diagnosis, distant metastases, surgery, and chemotherapy were independent prognostic factors.

Keywords: Hepatoid adenocarcinoma; SEER; incidence; clinical characteristics; prognosis

Submitted Feb 21, 2020. Accepted for publication Jul 17, 2020.

doi: $10.21037 /$ tcr-20-1126

View this article at: http://dx.doi.org/10.21037/tcr-20-1126

\section{Introduction}

Hepatoid adenocarcinoma (HAC) is defined as an extrahepatic adenocarcinoma with hepatocyte differentiation, characterized by high malignancy, high invasiveness and poor prognosis. The tumor composition can be simple HAC or HAC with typical acinar or papillary adenocarcinoma, signet ring cell or neuroendocrine carcinoma (1). Although most HACs are associated with elevated $\alpha$-fetoprotein (AFP) levels, the current pathological diagnosis is based on morphological features, regardless of serum AFP levels or immumohistochemical AFP staining (2). HAC occurs mostly in the lung, gastrointestinal tract and digestive organs, and the current treatment methods 


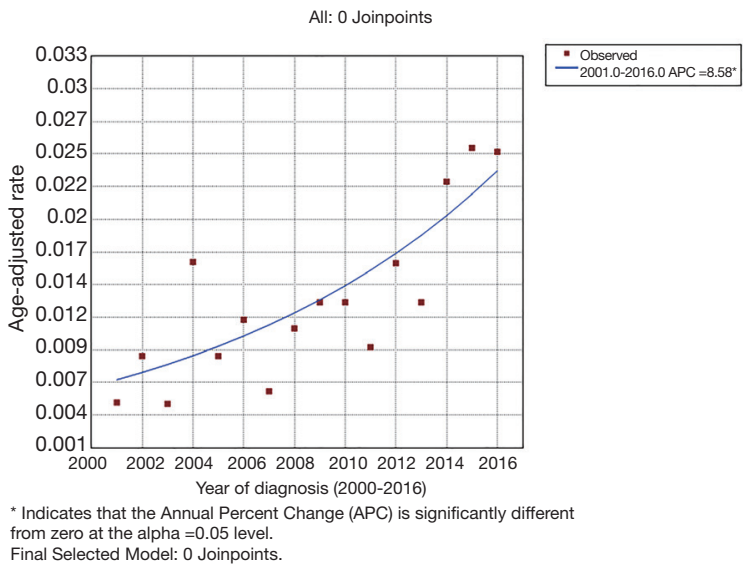

Figure 1 The incidence of hepatoid adenocarcinoma from 2000 to 2016.

depend on the primary site, as are other common types of adenocarcinoma.

The current researches on hepatoid adenocarcinoma are mostly case reports. This population-based study aimed to explore the clinical characteristics and prognostic factors of hepatoid adenocarcinoma, so as to provide an accurate prognostic basis for clinical diagnosis and treatment. We present the following article in accordance with the STROBE reporting checklist (available at http://dx.doi. org/10.21037/tcr-20-1126).

\section{Methods}

\section{Data acquisition}

We used SEER*Stat software (8.3.6) to acquire patient information. The prevalence rate of HAC was computed by the cohort of 258 patients from SEER 21 Regs LimitedField Research Data + Hurricane Katrina Impacted Louisiana Cases, Nov 2018 Sub [2000-2016] <Katrina/ Rita Population Adjustment>. We extracted another cohort from the SEER 18 Regs Custom Data (with additional treatment fields), Nov 2018 Sub (1975-2016 varying) by restricting the following: (I) ICD-O-3 Hist/behav of 8576/3: hepatoid adenocarcinoma; (II) known AJCC TNM staging; (III) complete information on surgery, radiotherapy or chemotherapy; (IV) active follow-up. Patients with unknown primary site were excluded because of possible bias in the survival analysis. Ultimately, 121 patients were used to estimate the survival and prognostic factors.

We extracted several variables from the selected cohort.
Clinical characteristics included age $(\leq 60,61-70,71-80$, or $\geq 81$ years), year of diagnosis (2004-2007, 2008-2011 or 2012-2015), race (white, black, or other), sex, marital status (single, married, or other), primary site (lung and bronchus, stomach, other gastrointestinal tract, pancreas, liver, gallbladder/bile duct or urogenital organ), grade, AJCC T stage (T0, T1, T2, T3, T4, or Tx), AJCC N stage (N0, $\mathrm{N} 1, \mathrm{~N} 2$, or Nx) and AJCC M stage (M0, M1). Treatment included surgery (yes or autopsy ONLY), radiotherapy (yes or no/unknown) and chemotherapy (yes or no/unknown). The primary endpoint was overall survival (OS).

\section{Statistical analysis}

The incidence was computed by the Joinpoint Regression Program (Version 4.7.0.0). We used IBM SPSS Statistics for survival analysis. The 1-, 3-, 6-, 12- and 36-month OS was estimated using the life table method. Univariate analysis was performed using the Kaplan-Meier method, and the log-rank test was used for statistical comparisons. Multivariate analysis was further performed to determine independent prognostic factors with the Cox proportional hazards model. $\mathrm{P}<0.05$ was considered statistically significant.

\section{Results}

\section{Incidence}

A total of 258 eligible patients were included in the study to compute the incidence. The overall incidence of HAC between 2000 and 2016 was 0.014 per 100,000 people (95\% CI: 0.012 to 0.015 ). The incidence in 2000 was 0 , and the age-adjusted incidence in 2001 was 0.005 , rising in volatility to 0.025 in 2016 . The final selected model fitted by joinpoint regression analysis is a gently ascending curve with no joinpoint (as shown in Figure 1).

\section{Clinical manifestations and treatment}

A total of 121 patients were obtained, and their clinical manifestations and treatments are shown in Table 1. Most of the patients were elderly, with $66.1 \%$ over 60 years old. There were 60 cases of HAC in the lungs, 23 in the digestive tract, 27 in the digestive organs, and 11 in the urinary or reproductive systems. Seven cases of HAC occurred in the liver, and it is particularly important to distinguish HAC from primary liver cancer. Of the 55 patients with known pathological grade, 51 had high-grade 
Table 1 Characteristics of hepatoid adenocarcinoma patients from the SEER Database

\begin{tabular}{|c|c|c|}
\hline Characteristics & \multicolumn{2}{|c|}{ Total $(n=121)$} \\
\hline \multicolumn{3}{|l|}{ Age, years } \\
\hline$\leq 60$ & 41 & $33.9 \%$ \\
\hline $61-70$ & 37 & $30.6 \%$ \\
\hline$\geq 81$ & 20 & $16.5 \%$ \\
\hline \multicolumn{3}{|l|}{ Race } \\
\hline White & 97 & $80.2 \%$ \\
\hline Black & 14 & $11.6 \%$ \\
\hline Male & 62 & $51.2 \%$ \\
\hline Female & 59 & $48.8 \%$ \\
\hline \multicolumn{3}{|l|}{ Marital status } \\
\hline Single & 27 & $22.3 \%$ \\
\hline Married & 60 & $49.6 \%$ \\
\hline Other & 34 & $28.1 \%$ \\
\hline \multicolumn{3}{|l|}{ Year of diagnosis } \\
\hline 2004-2007 & 27 & $22.3 \%$ \\
\hline Other gastrointestinal tract & 6 & $5.0 \%$ \\
\hline Pancreas & 12 & $9.9 \%$ \\
\hline Liver & 7 & $5.8 \%$ \\
\hline Gallbladder or bile duct & 8 & $6.6 \%$ \\
\hline Urogenital organ & 11 & $9.1 \%$ \\
\hline Grade & & $0.0 \%$ \\
\hline$I-I I$ & 4 & $3.3 \%$ \\
\hline III-IV & 51 & $42.1 \%$ \\
\hline Unknown & 66 & $54.5 \%$ \\
\hline
\end{tabular}

Table 1 (continued) 
Table 1 (continued)

\begin{tabular}{|c|c|c|}
\hline Characteristics & \multicolumn{2}{|c|}{ Total $(n=121)$} \\
\hline \multicolumn{3}{|l|}{ AJCC T } \\
\hline TO & 2 & $1.7 \%$ \\
\hline $\mathrm{T} 1$ & 19 & $15.7 \%$ \\
\hline T3 & 21 & $17.4 \%$ \\
\hline $\mathrm{T} 4$ & 36 & $29.8 \%$ \\
\hline Tx & 18 & $14.9 \%$ \\
\hline \multicolumn{3}{|l|}{ AJCC N } \\
\hline N2 & 19 & $15.7 \%$ \\
\hline N3 & 14 & $11.6 \%$ \\
\hline $\mathrm{Nx}$ & 17 & $14.0 \%$ \\
\hline \multicolumn{3}{|l|}{ AJCC M } \\
\hline MO & 49 & $40.5 \%$ \\
\hline M1 & 72 & $59.5 \%$ \\
\hline \multicolumn{3}{|l|}{ Surgery } \\
\hline No/autopsy only & 87 & $71.9 \%$ \\
\hline No/unknown & 66 & $54.5 \%$ \\
\hline Yes & 55 & $45.5 \%$ \\
\hline
\end{tabular}

adenocarcinoma. According to the AJCC TNM staging, $50.4 \%$ of patients had lymph node metastasis, and $59.5 \%$ had distant metastasis. In terms of treatment, 34 patients underwent surgery, and 18 underwent radiotherapy or chemotherapy after surgery. Seventeen patients received radiotherapy alone, and 16 received chemotherapy alone.

\section{Univariate and multivariate survival analyses}

The 1-, 3-, and 6-month survival rates for the cohort were
$68.6 \%, 59.5 \%$, and $45.4 \%$, respectively. The 1 -year survival rate was $35.0 \%$, while the 3 - and 5 -year survival rates were only $16.9 \%$. The median survival time of these patients was 5 months (95\% CI: 2.97 to 7.03 months).

Univariate analysis was performed using the following variables to determine the prognostic factors for OS: age, race, sex, marital status, year of diagnosis, primary site, pathological grade, AJCC TNM stage, and surgery, radiotherapy and chemotherapy acceptance. The results indicated that age, primary site, pathological grade, lymph 
node metastasis status, distant metastasis status, and surgery and chemotherapy acceptance were associated with the prognosis of these patients (as shown in Table 2).

Cox multivariate analysis was performed for factors with $\mathrm{P} \leq 0.05$ in univariate analysis, and the results showed that age, distant metastasis status, and surgery and chemotherapy acceptance were independent prognostic factors (as shown in Table 2). Kaplan-Meier survival curves were stratified by age, distant metastasis status, and surgery and chemotherapy acceptance, as shown in Figure 2. For patients $\leq 60,61-70$, $71-80$, and $\geq 81$ years old, the 6 -month OS rates were $65.7 \%, 35.1 \%, 39.1 \%$, and $30.0 \%$, and the 1 -year survival rates were $55.5 \%, 24.3 \%, 26.1 \%$, and $24.0 \%$, respectively. Patients without distant metastasis had a much better prognosis than those with distant metastasis, with a 1-year survival rate of $61 \%$ versus $17.1 \%$. According to the statistical results, surgery and chemotherapy acceptance were independent prognostic factors for $\mathrm{HAC}(\mathrm{HR}=0.268$; 95\% CI: 0.099 to $0.723, \mathrm{P}=0.009$; $\mathrm{HR}=0.463 ; 95 \% \mathrm{CI}$ : 0.282 to $0.760, \mathrm{P}=0.002)$. Of the patients who underwent surgery, $55.9 \%$ were primary in the gastrointestinal tract and digestive organs, and $26.5 \%$ were primary in the urogenital system. The one-year OS rate in these patients was $76.2 \%$, which was much higher than the $18.7 \%$ in inoperable patients.

\section{Discussion}

In the current study, we used a large data set from the SEER database to investigate the incidence and prognostic factors of HAC. The age-adjusted rate of HAC between 2000 and 2016 was 0.014 per 100,000 people (95\% CI: 0.012 to 0.015$)$. During this period, the age-adjusted incidence of HAC rose gently, with no joinpoint. This rising trend may be due to the increased awareness of HAC. Ishikura first proposed the term hepatoid adenocarcinoma in 1985 (3). The diagnosis of HAC is based on pathological morphological features. Previously, there were few studies on HAC, which made the differential diagnosis more difficult. With the increasing cognition of the special clinical and pathological features of HAC, the diagnosis rate of HAC also gradually increased $(4,5)$.

Through survival analysis, we found that HAC had a poor prognosis with a 1 -year survival rate of $35.0 \%$. The 3 and 5 -year survival rates were both only $16.9 \%$ (95\% CI: 2.97 to 7.03 months). The median survival time of these patients was 5 months. The OS of HAC in our study was poor compared to that in historical studies. In the review by
Metzgeroth et al., the 1-year OS rate of 83 HAC patients was $55 \%$, and the median survival time was 11 months (range, 0.1-102) (6). Grossman et al. reviewed 14 pulmonary HAC patients with survival information, and the 1-year survival rate was $50 \%$ (7). Zeng et al. analyzed the survival of 122 gastric HAC patients from their center and historical studies, and the 1-, 3-, and 5-year disease-specific survival rates were $62.8 \%, 37.2 \%$, and $29.2 \%$, respectively (8). Due to the rarity of HAC, literature reviews of previously published case reports constituted the bulk of the data in the above studies leading to inevitable publication bias. The limited sample size may be another reason for the different prognoses in our study.

In our study, the most common HAC sites were the lung (49.6\%), stomach (14\%), pancreas (9.9\%), urogenital organ $(9.1 \%)$, and gallbladder or bile duct (6.6\%). Interestingly, the anatomic incidence of HAC was quite different from that of the case report groups. Metzgeroth reviewed 261 previously published case reports of HAC before 2010, and the results suggested that the most common HAC locations were the stomach $(63 \%)$, ovaries (10\%), lung (5\%), gallbladder (4\%), pancreas (4\%), and uterus (4\%) (6). Although the proportion of HAC primary sites is still controversial, our research suggests that in recent years, pulmonary HAC has become the most common site, prompting clinicians to pay more attention to it. Moreover, our study shows that there was no significant correlation between prognosis and tumor location. In the studies of Metzgeroth and Hoshida, the prognosis of patients with HAC originating in the ovary and endometrium was slightly better than that of other patients, but the difference was not statistically significant due to the small sample size $(6,9)$.

Through multivariate analysis, we found that age at diagnosis, distant metastasis status, surgery and chemotherapy acceptance were independent prognostic factors for HAC. These results could help doctors choose the proper treatment for HAC patients.

Hepatoid adenocarcinoma is more likely to occur in elderly patients, with $66.1 \%$ of patients older than 60 years. The median age was 66 (range, 31-85) years, similar to the previously reported 65 years (6). The previously reported median ages of gastric HAC were 65.5 and 66 years $(10,11)$. In addition, our study is the first to find that patients younger than 60 years have better outcomes, with a 1-year survival rate as high as $55.5 \%$.

Our study found that $59.5 \%$ of HAC patients had distant metastases (mainly in the liver and lung) at the time of diagnosis. After analysis, the prognosis of patients 
Table 2 Univariate and multivariate COX regression for hepatoid adenocarcinoma

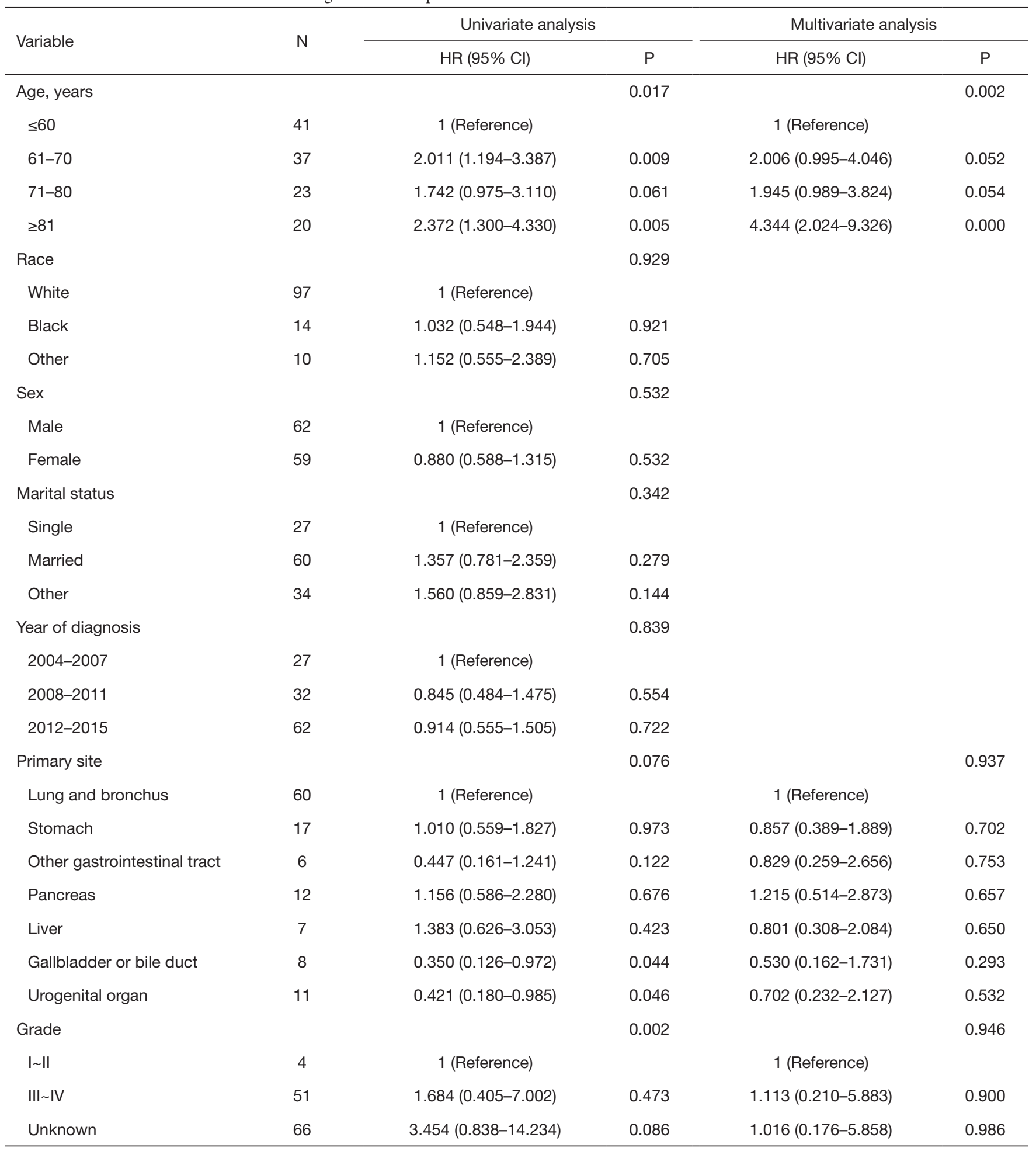

Table 2 (continued) 
Table 2 (continued)

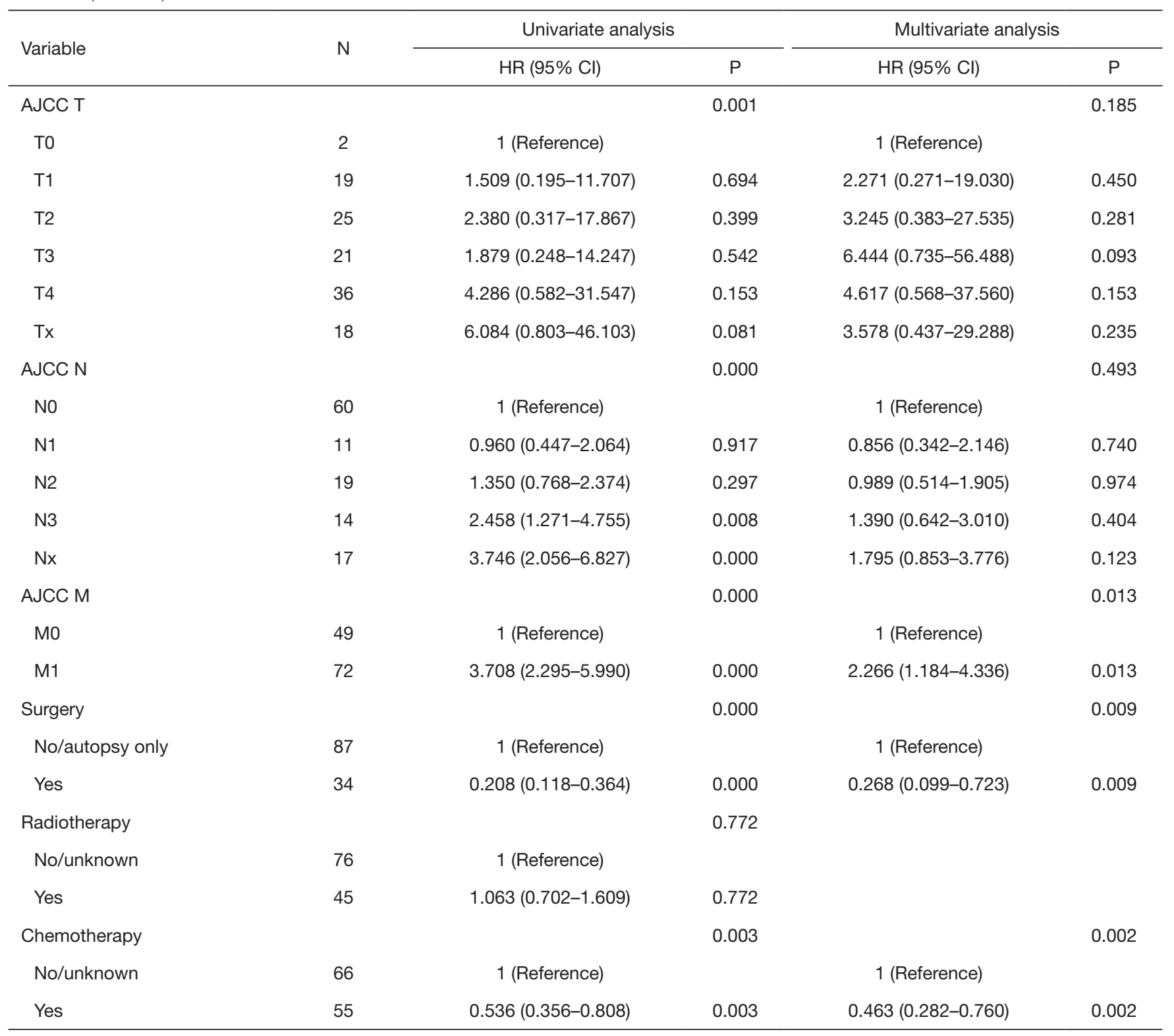

$\mathrm{Cl}$, confidence interval.

with distant metastasis was significantly worse than that of patients without distant metastasis. There was no significant survival difference in lymph node staging. Historical studies have found that both gastric and intestinal HAC pTNM staging were independent prognostic factors, but no subgroup analysis of $\mathrm{T}, \mathrm{N}$, and $\mathrm{M}$ staging was performed (12-15). Yang et al. reported three pancreatic HAC cases and reviewed an additional 31 cases in the literature. The results suggested that lymph node and distant metastases were associated with worse disease-specific survival $(\mathrm{P}=0.002$ for both) (16).

Currently, the treatment of HAC is determined by the primary site, as are other common types of adenocarcinoma. Patients with local tumors undergo surgical treatment. Others with lymph node metastasis or distant metastasis undergo radiation or/and chemotherapy. Our study found that surgery and chemotherapy acceptance were independent factors for improved survival. Zhang et al. 
A

Survival of hepatoid adenocarcinoma stratified by age

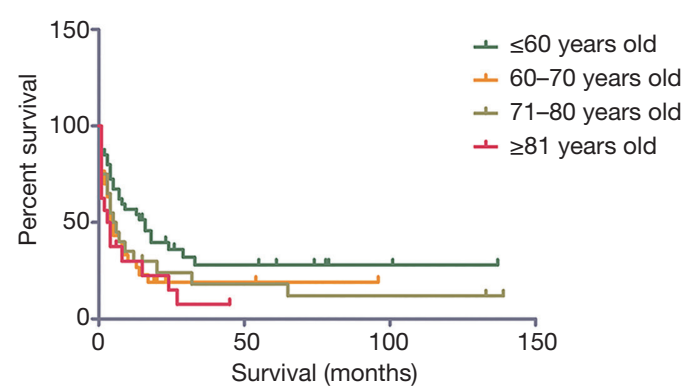

C

C Survival of hepatoid adenocarcinoma

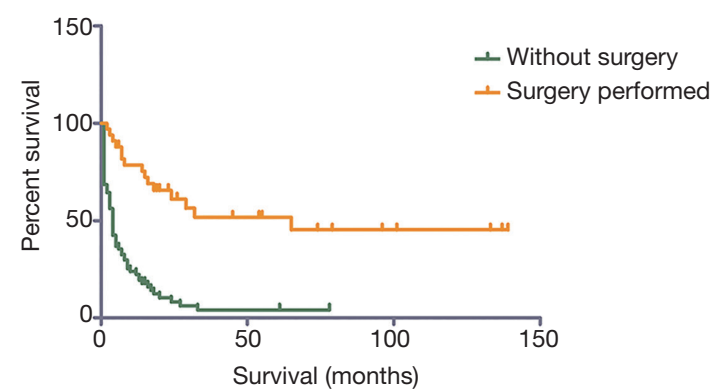

B
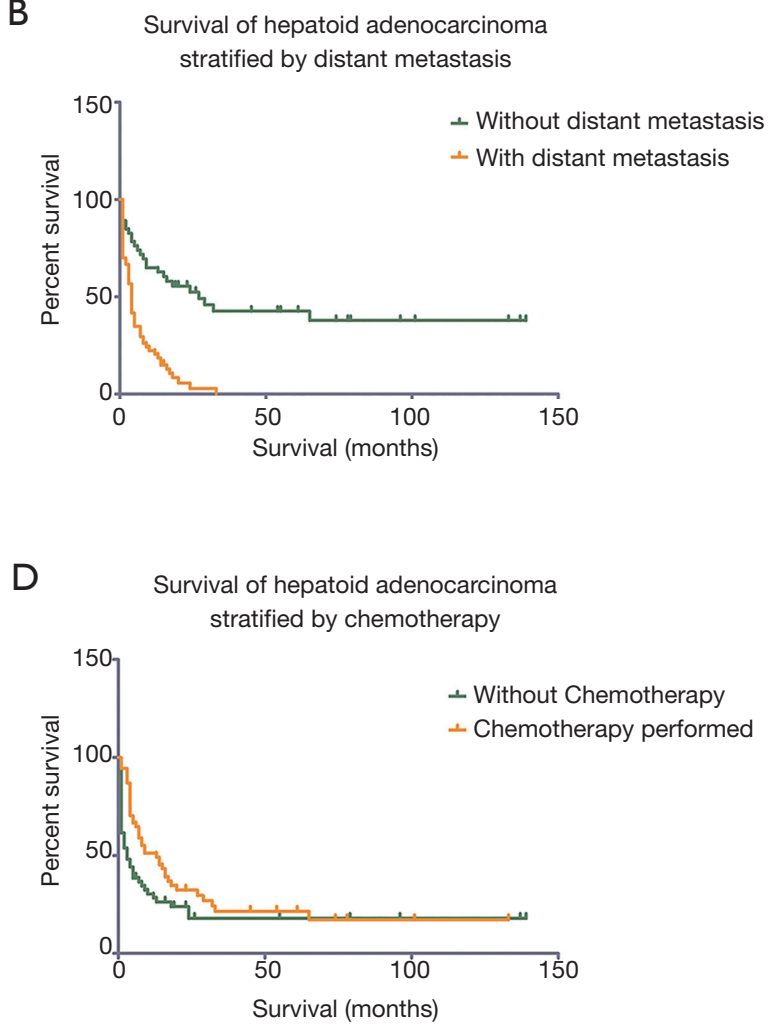

Figure 2 Kaplan-Meier survival curves of overall survival in hepatoid adenocarcinoma. (A) Survival of hepatoid adenocarcinoma stratified by age; (B) Survival of hepatoid adenocarcinoma stratified by distant metastasis status; (C) Survival of hepatoid adenocarcinoma stratified by surgery acceptance; (D) Survival of hepatoid adenocarcinoma stratified by chemotherapy acceptance.

also proposed that chemotherapy was an independent prognostic factor in 20 cases of gastric HAC (13). 5-FUbased chemotherapy was found to benefit survival in patients with gastric HAC (9). Another study found that among 18 cases of HAC with distant metastasis, a cisplatinbased regimen showed better efficacy than an irinotecan, gemcitabine, oxaliplatin or 5 -FU regimen, with $75 \%$ of the patients having clinically observed remission (17). In addition, patients undergoing neoadjuvant therapy were found to have a higher survival rate than those only undergoing postoperative adjuvant therapy. Therefore, for those patients diagnosed with an advanced pTNM stage, neoadjuvant therapy is strongly recommended to promote patient survival (8). Our study found that there was no significant difference in survival between patients with radiotherapy and those without radiotherapy. This finding suggests that HAC may be a kind of tumor with radiation resistance, and the molecular mechanism of which still needs further study.

Molecular targeted therapy may offer hope to patients with specific genetic mutations. In studies of targeted therapy for pulmonary HAC patients, an ALK gene rearranged patient was treated with crizotinib, and the tumor shrank radiologically in the first 2 months. However, after 6 months, the tumor showed imaging progression (18). Basse Victor reported a case of durvalumab anti-PD-L1 therapy. Although the PD-L1 status was negative, the patient still had a partial response to immunotherapy (19). This result may be related to the patient's mismatch-repair status. Mismatch repair status patients without effective treatment may benefit from PD-1 blockade (20). Ramucirumab, a monoclonal antibody for VEGF receptor-2, showed a significant clinical effect in a case of chemotherapy-resistant gastric HAC (21). According to the current studies, it can be speculated that targeted drugs can be used as adjuvant therapy to control the development of tumors but may need to be combined with other therapies to completely control tumor progression.

The limitations of this work include the following: (I) 
the lack of data on immunohistochemistry and serum tumor markers, such as AFP, which may be key prognostic factors; (II) the lack of some traditional treatment information in the SEER database, such as chemotherapy regimens and surgical margin status; (III) the unavailability of targeted therapy information in the SEER database; and (IV) the relatively small number of patients, which is insufficient to fully explain the occurrence, treatment and prognosis of this rare tumor.

\section{Conclusions}

This study suggests that HAC is a rare malignant tumor with a gradually increasing incidence and a poor prognosis. The most common sites were the lung (49.6\%), stomach (14\%), pancreas $(9.9 \%)$, urogenital organ $(9.1 \%)$, and gallbladder or bile duct (6.6\%). Age at diagnosis, distant metastases, surgery, and chemotherapy were independent prognostic factors.

\section{Acknowledgments}

Funding: None.

\section{Footnote}

Reporting Checklist: The authors have completed the STROBE reporting checklist. Available at http://dx.doi. org/10.21037/tcr-20-1126

Conflicts of Interest: Both authors have completed the ICMJE uniform disclosure form (available at http://dx.doi. org/10.21037/tcr-20-1126). The authors have no conflicts of interest to declare.

Ethical Statement: The authors are accountable for all aspects of the work in ensuring that questions related to the accuracy or integrity of any part of the work are appropriately investigated and resolved.

Open Access Statement: This is an Open Access article distributed in accordance with the Creative Commons Attribution-NonCommercial-NoDerivs 4.0 International License (CC BY-NC-ND 4.0), which permits the noncommercial replication and distribution of the article with the strict proviso that no changes or edits are made and the original work is properly cited (including links to both the formal publication through the relevant DOI and the license). See: https://creativecommons.org/licenses/by-nc-nd/4.0/.

\section{References}

1. Haninger DM, Kloecker GH, Bousamra Ii M, et al. Hepatoid adenocarcinoma of the lung: report of five cases and review of the literature. Mod Pathol 2014;27:535-42.

2. Xie Y, Zhao Z, Li P, et al. Hepatoid adenocarcinoma of the stomach is a special and easily misdiagnosed or missed diagnosed subtype of gastric cancer with poor prognosis but curative for patients of $\mathrm{pN} 0 / 1$ : the experience of a single center. Int J Clin Exp Med 2015;8:6762-772.

3. Ishikura H, Fukasawa Y, Ogasawara K, et al. An AFPproducing gastric carcinoma with features of hepatic differentiation. A case report. Cancer 1985;56:840-8.

4. Chen EB, Wei YC, Liu HN, et al. Hepatoid Adenocarcinoma of Stomach: Emphasis on the Clinical Relationship with Alpha-Fetoprotein-Positive Gastric Cancer. Biomed Res Int 2019;2019:6710428.

5. Liu X, Sheng W, Wang Y. An analysis of clinicopathological features and prognosis by comparing hepatoid adenocarcinoma of the stomach with AFPproducing gastric cancer. J Surg Oncol 2012;106:299-303.

6. Metzgeroth G, Ströbel P, Baumbusch T, et al. Hepatoid adenocarcinoma - review of the literature illustrated by a rare case originating in the peritoneal cavity. Onkologie 2010;33:263-9.

7. Grossman K, Beasley MB, Braman SS. Hepatoid adenocarcinoma of the lung: Review of a rare form of lung cancer. Respir Med 2016;119:175-9.

8. Zeng XY, Yin YP, Xiao H, et al. Clinicopathological Characteristics and Prognosis of Hepatoid Adenocarcinoma of the Stomach: Evaluation of a Pooled Case Series. Curr Med Sci 2018;38:1054-61.

9. Hoshida Y, Nagakawa T, Mano S, et al. Hepatoid adenocarcinoma of the endometrium associated with alpha-fetoprotein production. Int J Gynecol Pathol 1996;15:266-9.

10. Lin CY, Yeh HC, Hsu CM, et al. Clinicopathologial features of gastric hepatoid adenocarcinoma. Biomed J 2015;38:65-9.

11. Chen EB, Wei YC, Liu HN, et al. Hepatoid Adenocarcinoma of Stomach: Emphasis on the Clinical Relationship with Alpha-Fetoprotein-Positive Gastric Cancer. Biomed Res Int 2019;2019:6710428.

12. Zeng X, Zhang P, Xiao H, et al. Clinicopathological features and prognosis of intestinal hepatoid adenocarcinoma: evaluation of a pooled case series. Oncotarget 2017;9:2715-25.

13. Zhang JF, Shi SS, Shao YF, et al. Clinicopathological and 
prognostic features of hepatoid adenocarcinoma of the stomach. Chin Med J (Engl) 2011;124:1470-6.

14. Qu BG, Bi WM, Qu BT, et al. PRISMA-Compliant Article: Clinical Characteristics and Factors Influencing Prognosis of Patients With Hepatoid Adenocarcinoma of the Stomach in China. Medicine (Baltimore) 2016;95:e3399.

15. Yang J, Wang R, Zhang W, et al. Clinicopathological and prognostic characteristics of hepatoid adenocarcinoma of the stomach. Gastroenterol Res Pract 2014;2014:140587.

16. Yang C, Sun L, Lai JZ, et al. Primary Hepatoid Carcinoma of the Pancreas: A Clinicopathological Study of 3 Cases With Review of Additional 31 Cases in the Literature. Int J Surg Pathol 2019;27:28-42.

17. Simmet V, Noblecourt M, Lizée T, et al. Chemotherapy of metastatic hepatoid adenocarcinoma: Literature review and two case reports with cisplatin etoposide. Oncol Lett 2018;15:48-54.

18. Khozin S, Roth MJ, Rajan A, et al. Hepatoid carcinoma of the lung with anaplastic lymphoma kinase gene rearrangement. J Thorac Oncol 2012;7:e29-e31.

19. Basse V, Schick U, Guéguen P, et al. A Mismatch RepairDeficient Hepatoid Adenocarcinoma of the Lung Responding to Anti-PD-L1 Durvalumab Therapy Despite no PD-L1 Expression. J Thorac Oncol 2018;13:e120-e2.

20. Le DT, Uram JN, Wang H, et al. PD-1 Blockade in Tumors with Mismatch-Repair Deficiency. N Engl J Med 2015;372:2509-20.

21. Arakawa Y, Tamura M, Aiba K, et al. Significant response to ramucirumab monotherapy in chemotherapy-resistant recurrent alpha-fetoprotein-producing gastric cancer: A case report. Oncol Lett 2017;14:3039-42.
Cite this article as: Wang W, Li G. Incidence and prognostic factors of hepatoid adenocarcinoma: a population-based analysis. Transl Cancer Res 2020;9(9):5401-5410. doi: 10.21037/ tcr-20-1126 